\title{
Papel del estrés en la etiopatogenia de la Enfermedad Periodontal
}

\author{
BARBIERI PETRELLI G * \\ MATEOS RAMÍREZ L ** \\ BASCONES MARTÍNEZ ***
}

\begin{abstract}
Barbieri Petrelli G, Mateos Ramírez L, Bascones Martínez A. Papel del estrés en la etiopatogenia de la Enfermedad Periodontal. Av Periodon Implantol. 2003; 15, 2: 77-86.
\end{abstract}

\begin{abstract}
RESUMEN
En el carácter crónico de la periodontitis está determinada la influencia de condiciones o factores de riesgo en la progresión de este cuadro patológico. Entre éstas se encuentran diabetes, tabaco, desórdenes genéticos, etc. Recientemente, diversos estudios han intentado relacionar el estrés psicológico a la prevalencia y progresión de la EP, con distintos resultados en función de la población estudiada, así como de la capacidad de aislar este posible factor de riesgo de otros que pudieran influir directa o indirectamente en los resultados. Diversos trabajos han estudiado las bases moleculares y celulares de la interacción entre el sistema inmune y el sistema nervioso central (SNC), relación que interviene directamente en el mecanismo homeostático del estrés en aspectos tanto inmunológicos como de comportamiento. El objetivo de este artículo es realizar una revisión bibliográfica sobre la asociación etiopatogénica del estrés psicológico y la enfermedad periodontal, realizando un análisis de la evidencia científica disponible en la relación de ambas entidades.
\end{abstract}

\section{PALABRAS CLAVE}

Estrés, enfermedad periodontal, etiología, patogénesis.

\section{INTRODUCCIÓN}

La periodontitis, una de las enfermedades más antiguas y comunes del ser humano, se pensaba que era una consecuencia inevitable del envejecimiento. Sin embargo, ahora sabemos que no todas las personas y poblaciones tienen el mismo riesgo de desarrollar enfermedad periodontal. Numerosos estudios epidemiológicos y experimentales han ayudado a reconocer condiciones o factores de riesgo, permitiendo una mejor comprensión de por qué unos individuos son más susceptibles que otros. Entre estas condiciones o factores de riesgo encontramos la diabetes, el tabaco, los desórdenes genéticos, etc. Recientemente, diversos estudios han intentado relacionar el estrés psicológico a la prevalencia y progresión de la enfermedad periodontal, con diferentes resultados en función de la población estudiada, así como de la capacidad de aislar este posible factor de riesgo de otros que pudieran influir directa o indirectamente en los resultados.

\section{ESTRÉS}

El estrés es un proceso adaptativo y de emergencia que genera emociones, pero no es una emoción en sí mismo. Así, cada emoción está desencadenada por un tipo de situaciones muy específicas, mientras que el estrés se desencadena ante cualquier cambio en la estimulación o alteración de las rutinas cotidianas. Las

\footnotetext{
* Licenciado en Odontología.

** Licenciado en Odontología. Master Periodoncia e Implantología UCM. Práctica privada en Sevilla.

*** Catedrático de Medicina Bucal y Periodoncia. UCM.
} 
emociones se caracterizan por poseer una forma de afrontamiento propia de cada emoción, mientras que el estrés moviliza una amplísima gama de posibles afrontamientos.

El estrés no implica necesariamente que sean sucesos negativos; las situaciones positivas también pueden llevar a estrés. Los sucesos que dan lugar a estrés se llaman estresores porque son percibidos como amenazantes o desafiantes. El énfasis en la percepción significa que un suceso puede ser estresante para una persona y no para otra (1).

Hans Selye, un médico de Canadá, dedicó muchos años a examinar la respuesta del cuerpo a los estresores. Encontró que la respuesta de estrés, a lo que llamó síndrome general de adaptación, era sistemática y podría ser encuadrada en tres fases (Selye, 1976). Durante la primera fase (reacción de alarma), el cuerpo se prepara para enfrentarse con el estresor. Las hormonas adrenales tales como epinefrina, o adrenalina, son liberadas dentro del torrente sanguíneo, originando un incremento en la tasa cardíaca y respiratoria. Debido a que el mayor esfuerzo del cuerpo es focalizado para la preparación al enfrentamiento con el estresor, la resistencia del cuerpo se reduce temporalmente en otra parte. Si el estresor persiste, el cuerpo entra en la fase de resistencia. En esta fase, el cuerpo intenta conducir los recursos de emergencia a un nivel más normal, aunque está funcionando a un nivel más alto que lo normal. Si el estresor desaparece durante esta fase, el cuerpo retorna a un nivel de funcionamiento normal (1).

Al contrario, si la situación que produce estrés continúa, comienza la fase de agotamiento. Es en este punto donde el cuerpo comienza a agotarse, y reaparecen muchos de los síntomas de reacción de alarma (1).

\section{ESTRESORES}

Existen multitud de factores y situaciones que pueden conducir a un estado de estrés. En la mayoría de los cuestionarios se le pregunta al paciente por su trabajo, nivel socioeconómico, estado civil, familia, acontecimientos desafortunados en su vida, etc. Los cuestionarios pueden ser todo lo exhaustivos que el profesional desee.

Un grupo de estresores, que en muchas ocasiones no se tienen en cuenta, son aquellos consecuentes de la propia enfermedad periodontal: dolor, sangrado, gusto desagradable, halitosis, apariencia alterada de los dientes, movilidad, pérdida de dientes, etc. Además está el tratamiento (dolor, coste, etc). Todo esto puede ser un incentivo para el paciente con respecto al tratamiento (5).

\section{MECANISMO PATOGÉNICO DE LA ASOCIACIÓN ESTRÉS-ENFERMEDAD PERIODONTAL (Fig 1)}

\section{INFLUENCIA DEL ESTRÉS EN EL SISTEMA INMUNE}

Está ampliamente reconocido el impacto que produce el estrés agudo y crónico sobre el sistema inmune. El estrés agudo tiene un efecto estimulante sobre el sistema inmune, mientras que en el caso del estrés crónico (fundamentalmente con depresión) la regulación del sistema inmune podría estar disminuida. De todos modos, existe una variabilidad individual en la respuesta inmunológica al estrés (2).

El estrés es sugerido como un importante factor destructor de la regulación de la homeostasis entre microflora oral y el sistema inmune del hospedador (Genco 1992, Ainamo y Ainamo 1996, Seymour y cols 1993, Breivik y cols 1996).

Estudios psicoinmunológicos observaron que la defensa inmune contra antígenos se ve influida por la interacción entre comportamiento (estrés), sistema nervioso central y células del sistema inmune. Estas interacciones están mediadas por un "complejo inmuno-neuro-endocrino". La coordinación está basada en las uniones de las fibras neuronales del sistema nervioso autónomo y también en el eje hipotálamo - pituitaria - corteza suprarrenal, con cortisol de la corteza suprarrenal y citocinas (ej: IL- $1 \beta$, IL-6) jugando un importante papel como mediadores químicos (3) (Fig 2).

\section{INTERLEUCINA 1 BETA (IL-1 $\beta)$.}

Es una citocina pleiotrópica y uno de los activadores celulares más frecuentes y multifuncionales. Está producida principalmente por monocitos/macrófagos, células natural Killer y células B. Otras células como fibroblastos, queratinocitos y células endoteliales también pueden producirla. Además han sido vistas producidas por células de sistema nervioso central (SNC), junto a células de la microglia, astrocitos y células endoteliales, siendo discutidas como fuentes primarias (Besedovsky y del Rey 1996).

La actividad biológica de la IL-1 $\beta$ es extremadamente diversa, activando proteínas en fase aguda, prostaglandinas, otras citocinas, inducción de colágeno y 
síntesis de colagenasa, y reabsorción de calcio en el hueso. Además, en células inmunocompetentes se hallaron efectos estimuladores en la proliferación de linfocitos, inhibición de la síntesis de interferón (IFN) y una elevada producción de linfocinas de células T y de inmunoglobulinas de células plasmáticas. Esto conduce a una actividad proinflamatoria que regula y coordina el curso de la respuesta inmune pero podría también aumentar los efectos destructivos en sobrerreacciones.

La liberación de IL-1 $\beta$ es estimulada por numerosas sustancias, como linfocinas, otras citocinas, prostaglandina $\mathrm{E}_{2}\left(\mathrm{PG} \mathrm{E}_{2}\right)$, factores del complemento y bacterias o lipopolisacáridos (LPS) (Cavaillon y cols 1990, Cavaillon y Haeffner-Cavaillon 1990, Okusawa y cols 1987, Kunkel y Chensue 1985, Sisson y Dinarello 1988). En particular, la liberación inducida localmente por bacterias tiene especial interés en la enfermedad inflamatoria periodontal. Algunos estudios in vitro hallaron que la secreción de IL-1 $\beta$ podría estar estimulada por la activación de fagocitos mononucleares mediante LPS. También, la liberación de IL-1 $\beta$ podría estimular los fibroblastos gingivales para liberar cantidades sustanciales de PG E2 y enzimas colagenolíticas (Richards y Rutherford 1988, Meikle y cols 1989). Además, se ha registrado un efecto desmineralizante sobre el hueso alveolar (Tatakis 1993). Estos resultados in vitro fueron confirmados en algunos estudios clínicos, donde niveles elevados de IL-1 $\beta$ fueron detectados en el fluido crevicular, en el tejido conectivo gingival y en el suero sanguíneo de pacientes periodontales (Gemmell y cols 1997).

\section{INTERLEUCINA 6 (IL-6)}

Es una citocina multifuncional que puede ser secretada por varias células como monocitos/macrófagos, células T, fibroblastos, hepatocitos, células endoteliales y células neuronales (Van Snick 1990). Las principales fuentes en el organismo humano son las células T y B, fibroblastos y monocitos/macrófagos, con las células secretoras de IL-6 estimulándose las unas a las otras. Por ejemplo, la secreción de IL-6 de células T es dependiente de la estimulación por monocitos, mientras que los monocitos pueden producir IL$6 \mathrm{sin}$ la asistencia de otras células (Horii y cols 1988). La liberación de IL-6 es estimulada en la activación de monocitos/macrófagos por LPS, IL-1 $\beta$ y TNF- $\alpha$ (factor de necrosis tumoral alfa), mientras que las células T y B son estimuladas por la secreción de IL6 principalmente por mitógenos policlonales (Kishimoto 1989).

La actividad biológica de la IL-6 es altamente diversa, actuando en la diferenciación de células B en células plasmáticas, activación de células $\mathrm{T}$ y liberación de proteínas en fase aguda de los hepatocitos (Van Snick 1990).

\section{CORTISOL}

Es una hormona producida en la corteza suprarrenal, y uno de los glucocorticoides más importantes. Tiene un fuerte impacto sobre el metabolismo intermediario, induce un incremento en la concentración de azúcar en sangre, e influye en el metabolismo de las grasas. Además de estos efectos endocrinos, tiene importantes propiedades antiinflamatorias e inmunosupresoras, inhibiendo la formación de linfocitos e induciendo hiperplasia del tejido linfático (Sanan y Antón 1988). Como la producción de anticuerpos está inhibida simultáneamente, hay un marcado descenso en la defensa inmune humoral. Esto puede dar una oportunidad a las bacterias periodontales de proliferar y posiblemente invadir los tejidos, dando lugar, finalmente, a una respuesta inflamatoria más extensa.

La secreción del cortisol está regulada por el eje hipotálamo - pituitaria - corteza suprarrenal. La estimulación del hipotálamo induce secreción de hormona liberadora de corticotropina ( $\mathrm{CRH})$, que llega al lóbulo anterior de la glándula pituitaria, donde induce la secreción de la hormona adrenocorticotropa (ACTH). Ésta entra en la sangre periférica y estimula la corteza suprarrenal para secretar cortisol y otros esteroides. El cortisol se dirige directamente a la sangre periférica, vinculado a proteínas transportadoras (ej: transcortina) y tiene un efecto inhibitorio sobre la liberación de CRH y ACTH en forma de feedback negativo.

Influencias externas, como el estrés, tienen un claro efecto estimulante sobre el eje hipotálamo - pituitaria - corteza suprarrenal. Además, citocinas como IL-1 $\beta$ e IL-6 podrían estimular este eje directamente (Harbuz y cols 1992, Naito y cols 1990, Lyson y McCann 1991, Eskay y cols 1990), con IL-1 $\beta$ de linfocitos y macrófagos probablemente induciendo secreción en la región de la glándula pituitaria (Besedovsky y cols 1986).

Así, ambas vías estimuladoras conducen a la secreción de cortisol del eje adrenal (o suprarrenal) con los consecuentes efectos inmunosupresores.

Estudios experimentales mostraron que el uso de glucocorticoides sistémicos tiene un importante efecto inhibitorio en la producción de IL-12 en monocitos pero no sobre la IL-10 (Visser y cols 1998, Elenkov y cols 1996). Como la IL-12 regula la diferenciación de células T-helper a células Thl y la IL-10 estimula el desarrollo de células Th2, es asumido que el estrés induce una supresión selectiva de células Thl. Esto 
fue confirmado en algunos estudios clínicos con pacientes bajo estrés, donde se han encontrado concentraciones elevadas de citocinas IL-10 e IL-6, que son típicas de células Th2, en contraste con IFN- $\alpha$, en sangre periférica (Marshall y cols 1998, Song y cols 1999, Dobbin y cols 1991).

Pese a toda esta información, son todavía muy desconocidos los mecanismos por los que la defensa inmune contra anticuerpos o bacterias se ve influida por interacciones entre comportamiento (estrés), sistema nervioso central y células del sistema inmune.

El estrés mental y psicosocial puede resultar también en una activación del sistema nervioso autónomo y una secreción resultante de adrenalina y noradrenalina. Estas catecolaminas afectan entonces a las prostaglandinas y proteasas, que a su vez aumentan la destrucción periodontal (4). Por otro lado, disminuyen el fluido sanguíneo en la encía con la consecuente disminución de los elementos protectores frente a la infección en la zona.

El aumento de la habilidad para convivir con el estrés y reducir los efectos negativos mediante intervenciones psicológicas podría tener efectos beneficiosos sobre el sistema inmune (2).

\section{INFLUENCIA DEL ESTRÉS EN EL COMPORTAMIENTO}

Diversos autores defienden un modelo de afectación del estrés como causante de una variación en los hábitos del individuo que lo padece. Esto se manifiesta en la adquisición o aumento de factores de riesgo con afectación conocida sobre la enfermedad periodontal. Así, las personas estresadas parecen tener peores niveles de higiene oral, asociados a una falta de atención dental periódica, además de estar relacionados con consumo de tabaco. Esto puede estar unido a una dieta rica en productos grasos, que puede igualmente conducir a una depresión del sistema inmune por elevados niveles de cortisol.

\section{ESTUDIOS EN ANIMALES}

1. (Shapira y cols 1999-2000) $(7,8)$. Estudio en ratones sometidos a estrés físico (frío) y psicológico (aislamiento), además de tener un grupo control y un grupo con inducción de corticoesteroides. Este autor evidencia la distinta respuesta ante el estímulo lipopolisacárido de Porphiromonas gingivalis. En 1999 demostró un incremento en la migración leucocitaria así como en la producción de INF- $\gamma$ y TNF- $\alpha$ en los ratones sometidos a estrés respecto al grupo control cuando eran estimulados con patógenos periodontales (P.g.). En el año 2000, publicó un estudio que reflejaba la disminución del número de macrófagos reclutados ante estímulos (P.g.) en los ratones bajo situaciones de estrés. Sin embargo, la actividad de estos macrófagos (medida por la secreción de óxido nítrico) estaba aumentada en el grupo experimental.

En ambos trabajos, el estrés provocado por el aislamiento de los ratones desarrolló mayores alteraciones en la respuesta inmune que el provocado por el frío. Además, destaca la importancia de investigar los diversos mediadores celulares, debido a que, según sus experimentos, los corticoesteroides no son los únicos involucrados en la respuesta inmune diferente que sufre el organismo ante el estrés.

\section{ESTUDIOS EN SERES HUMANOS:}

1. (Shannon y cols 1969) (9). El estrés ambiental puede ser un factor importante en las exacerbaciones de los procesos de GUN y PUN. Shannon realizó un estudio comparando los niveles de corticoesteroides en orina (asociación a estrés) sobre una muestra de 474 individuos clasificados en 6 grupos según su grado de salud/enfermedad gingivo-periodontal. Los resultados mostraron una correlación estadísticamente significativa entre el grupo afectado de GUN y los niveles elevados de corticoesteroides en orina de esos pacientes.

2. (Freeman y cols 1993) (10). Realizaron un pequeño estudio prospectivo de adultos trabajadores en el que encontraron que una gran proporción de la varianza en la profundidad de las bolsas periodontales se podía explicar por el estrés ocupacional, junto con el hábito de fumar y la frecuencia de cepillado.

3. (Moss y cols 1996) (11). Estudio de casos-control sobre factores psicosociales y periodontitis adulta en el que se recogió información sobre depresión y nivel de estrés diario debido a factores como el trabajo, finanzas, familia, etc. Este estudio es valorado por su clara descripción al relacionar enfermedad periodontal con actividad del sistema inmune, estrés psicosocial y depresión. Está realizado en 71 individuos con enfermedad periodontal y 77 controles sin enfermedad, y se encontró que las personas con puntuaciones elevadas en la escala de depresión tenían casi tres veces más probabilidad de padecer enfermedad periodontal. También identifica líneas específicas de investigación para inquirir en cómo factores ambientales podrían influir en la enfermedad periodontal.

4. (Croucher y cols 1997) (12). Realizaron un estudio de 100 casos con al menos una bolsa con profundidad 


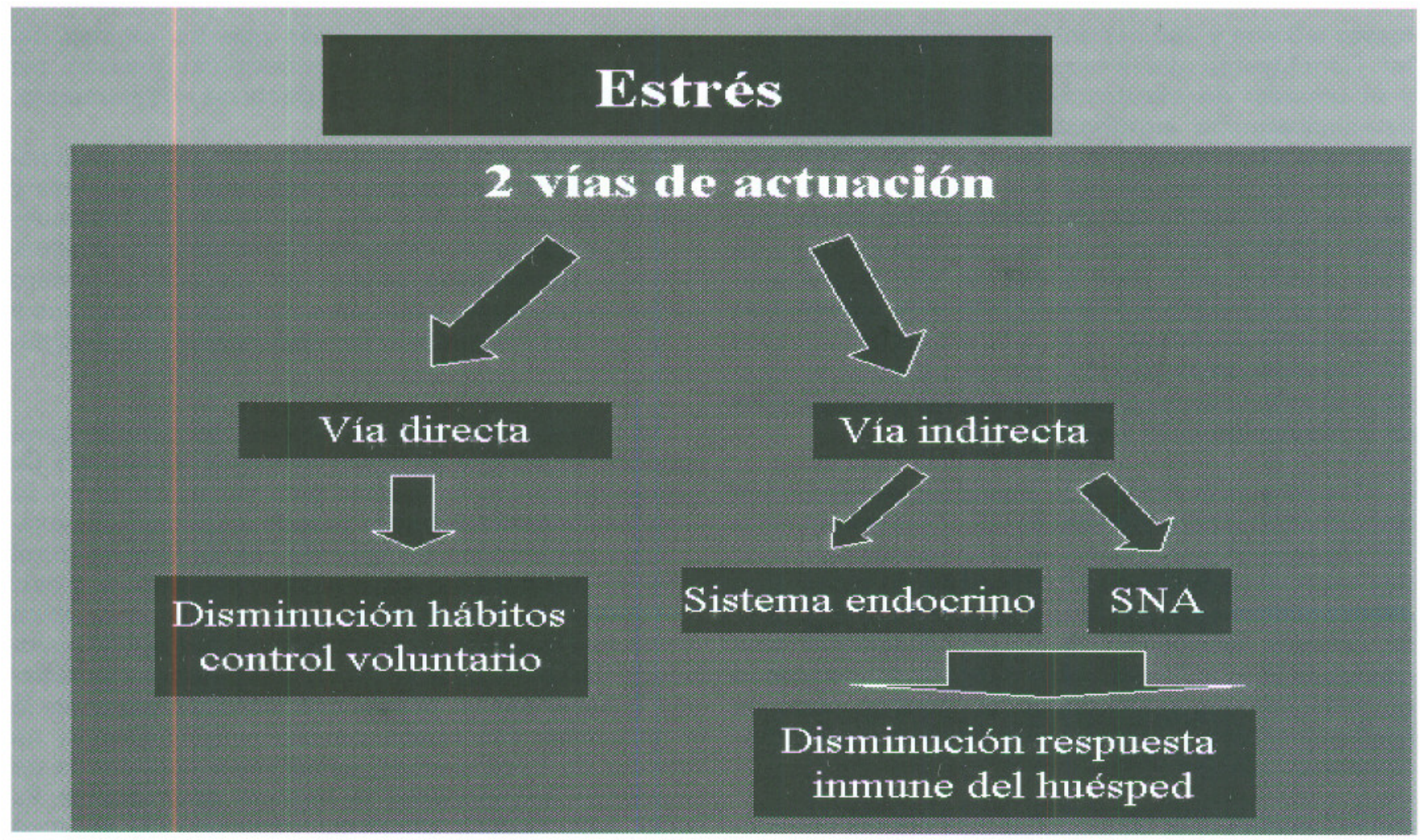

Fig. 1. Vías de actuación del mecanismo patogénico de la asociación de ambas entidades.

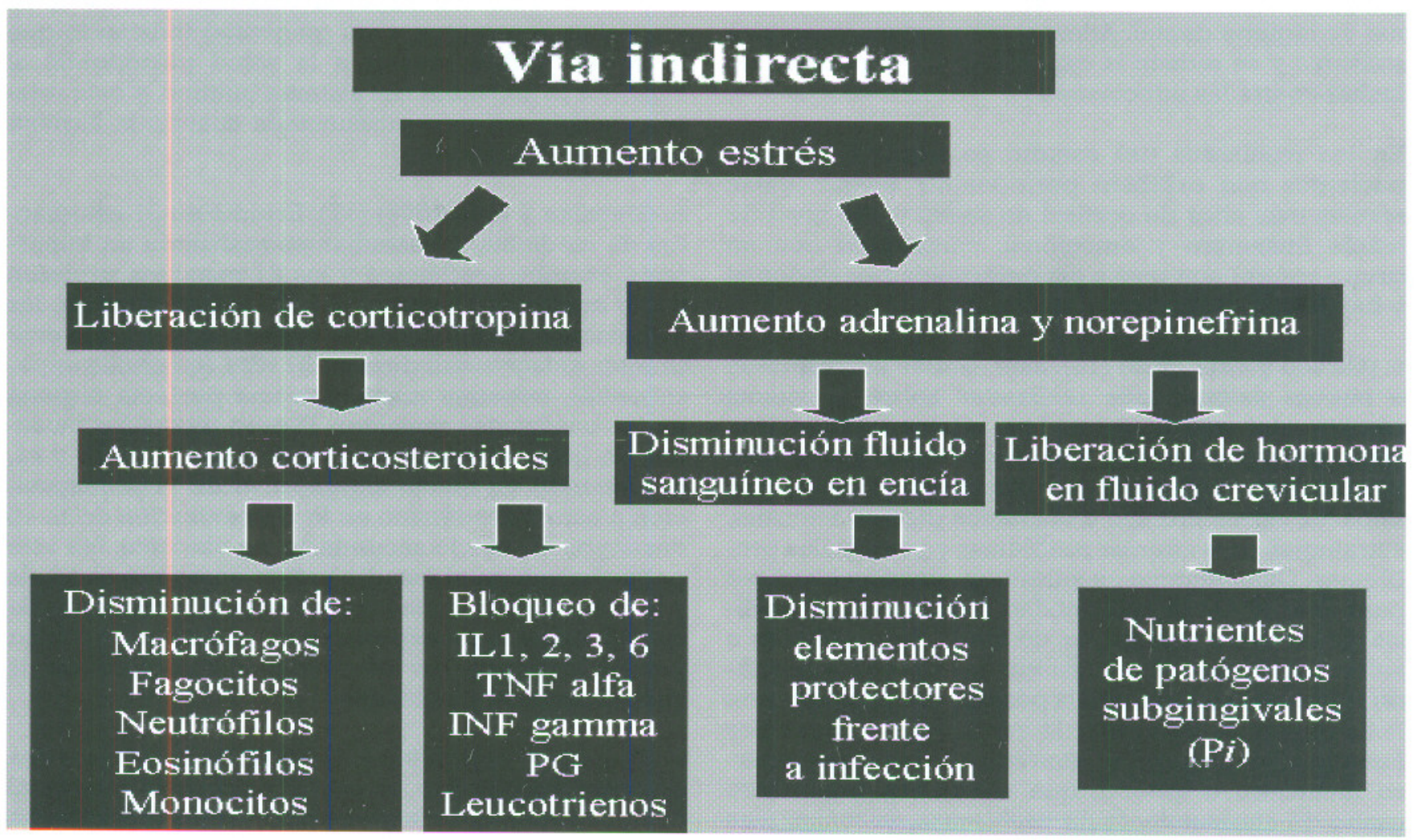

Fig. 2. Vía indirecta de la asociación etiopatogénica estrés-Enfernedad Periodontal. 
mayor a $5 \mathrm{~mm}$ y 100 controles sin bolsas periodontales. Concluyeron que la asociación entre periodontitis y el impacto de eventos negativos continúa siendo estadísticamente significativo incluso después de controlar otras variables como nivel de educación, número de dientes perdidos y estado matrimonial, pero no continúa siendo significativo después de controlar el hábito de fumar. Parece ser que el hecho de estar desempleado o de haber sufrido un acontecimiento desagradable está asociado a un aumento en el consumo de tabaco y un empeoramiento en los niveles de higiene oral, lo que corrobora el modelo conjunto de relación estrés-comportamiento, además de la afectación en el sistema inmune.

5. (Genco y cols 1998) (13). Evaluaron la asociación del estrés, angustia y conducta de afrontamiento con la enfermedad periodontal en 1426 sujetos, con edades comprendidas entre 25 y 74 años. Se recogieron características demográficas, médicas e historia dental. También consumición de tabaco y alcohol, al igual que placa supragingival, flora subgingival, sangrado gingival, cálculo, profundidad de sondaje, nivel de inserción clínica y pérdida ósea alveolar (radiográficamente).

Genco asegura que: "el estrés laboral supone una presión constante a largo plazo. Hemos visto que las personas que padecen mucha tensión o que se ven incapaces de solucionar un problema tienden a abandonar su higiene dental. Además, se producen cambios salivares y se reduce la capacidad del organismo de luchar contra las infecciones".

En los resultados del estudio se observó que los pacientes que exhibían periodontitis severa tenían niveles más altos de cortisol en saliva y alto nivel de estrés financiero y emocional; comparado con un grupo control con leve o sin enfermedad periodontal y bajo estrés financiero y emocional.

6. (Genco y cols 1999) (14). Este estudio corresponde a nuevas valoraciones realizadas sobre la misma muestra del año anterior. Los sujetos completaron cinco cuestionarios que valoraban rasgos y aptitudes psicológicas, incluyendo: acontecimientos vitales y su impacto, estrés crónico o esfuerzos diarios, angustia, estrategias para afrontar problemas y principales problemas. Clínicamente se valoraron diferentes índices para determinar la enfermedad periodontal. El análisis de los datos indicó que, de todos los problemas o esfuerzos diarios, sólo el estado financiero fue asociado significativamente con la pérdida de inserción y de hueso después de ajustar la edad, sexo y cigarrillos fumados. No obstante, sujetos con alto nivel de esfuerzo financiero o laboral, que mostraban importante grado de afrontamiento de problemas, no tenían más enfermedad periodontal que aquellos con bajo esfuerzo financiero, sugiriendo que los efectos del estrés en la enfermedad periodontal pueden ser moderados adecuando la conducta de afrontamiento.

Concluyeron que los resultados del estrés psicosocial asociado a esfuerzos o problemas financieros y angustia manifestada como depresión, son indicadores importantes de riesgo de enfermedad periodontal más severa para adultos en los que el género (masculino), tabaco, diabetes mellitus, B. forsythus y P. gingivalis son también indicadores de riesgo significativos.

7. (Deinzer y cols 1998-1999) $(15,16)$. Estudio prospectivo en el que se observó mayor frecuencia de deterioro severo de la salud gingival, en un grupo de sujetos, después de haber pasado un período de exámenes académicos, comparado con un grupo control que no experimentó estos exámenes. Deinzer y cols realizaron un experimento para valorar la relación entre estrés académico e inflamación gingival, valorando cambios en la IL-1 $\beta$, un componente del sistema inmune que se piensa que juega un papel en la destrucción del tejido periodontal. Utilizando un diseño de boca partida, estudiantes voluntarios de medicina abandonaron la higiene oral durante 21 días, para inducir gingivitis experimental en esos cuadrantes, mientras mantenían niveles altos de higiene oral en los otros dos cuadrantes. Se observó que los estudiantes tenían niveles elevados de IL- $1 \beta$ en ambas zonas (con y sin gingivitis) indicando que el estrés podría afectar a la salud periodontal al suprimir la actividad del sistema inmune, y esta relación podría ser más pronunciada cuando la higiene oral no es mantenida.

8. (Deinzer y cols 2000) (17). Estudio en 31 estudiantes de medicina. 18 estudiantes realizaron un importante examen académico y los 21 restantes sirvieron como controles. Desde el día del último examen, los estudiantes abandonaron la higiene oral en dos cuadrantes antagonistas durante 21 días (gingivitis experimental), mientras mantenían una perfecta higiene en los cuadrantes restantes. Fueron recogidas muestras de fluido crevicular en los días $0,5,8,15,18$ y 21 . Se encontró un efecto significativo de la preexposición a estrés académico en la concentración de IL-1 $\beta$ crevicular. El efecto secundario, no obstante, fue más pequeño que en el estudio previo en el que el estrés y la placa fueron presentados concomitantemente. Se concluye que la preexposición al estrés podría alterar persistentemente los efectos inmunológicos de la microflora en el periodonto.

9. (Deizner y cols 2001) (18). Mientras parece ser una creencia común que el estrés psicosocial afecta al comportamiento de higiene oral, esta suposición ha sido raramente probada. Este estudio sí intenta anali- 
zar los efectos del estrés en la higiene oral. 16 pares de estudiantes de medicina (uno participa en un importante examen académico y el otro no, es decir, sin estrés académico). Se realizó una limpieza profesional al inicio del estudio (baseline). En el último día de exámenes, los estudiantes respondieron cuestionarios sobre higiene oral durante los exámenes y se les realizó una segunda exploración dental (de la que no tenían conocimiento previo). En el último día de exámenes (aproximadamente 6 semanas tras limpieza profesional), los controles mostraron más superficies libres de placa. Los estudiantes admitieron una disminución en la minuciosidad pero no en la frecuencia de higiene oral.

10. (Axtelius y cols 1998) (19). Dos grupos de pacientes fueron comparados: (a) buena respuesta al tratamiento $(n=11),(b)$ peor respuesta al tratamiento $(n=11)$. Factores somáticos y psicológicos fueron descritos al obtener información de encuestas y tests psicológicos. El segundo grupo mostraba mayor estrés psicosocial y personalidad más pasiva y dependiente. El primer grupo mostraba personalidad más rígida y posiblemente una menor situación psicosocial de estrés en el pasado. El artículo destaca la posible contribución del estrés a la resistencia al tratamiento periodontal. Los autores concluyen que es importante valorar la personalidad y estado psicológico del paciente a la hora de valorar el pronóstico del tratamiento periodontal.

11. (Shizukuishi y cols 1998) (20). Estudio que relaciona el estilo de vida y estatus de salud periodontal de los trabajadores de una fábrica japonesa. En una revisión de salud anual, el estado de salud periodontal fue calculado usando el Índice Periodontal Comunitario (CPI). La información sobre el estilo de vida fue obtenida por cuestionarios. Análisis de dos variables y multivariado fueron utilizados para examinar la relación entre estilo de vida, cuidado oral y dos indicadores del estado de salud periodontal.

En el análisis de dos variables, las variables significativas fueron edad, tabaco, alcohol, frecuencia de cepillado, método de cepillado y uso de suplementos interdentales. Cantidad de tabaco o alcohol fue asociada con el estatus de salud periodontal.

Los datos de este estudio indican que el estilo de vida (incluyendo tabaco y cuidado oral insuficiente) tiene una asociación independiente con la enfermedad periodontal.

12. (Vanderas y cols 1998) (21). Estudio de gingivitis en 314 jóvenes entre 6 y 8 años. Los niveles de catecolaminas urinarias (epinefrina, norepinefrina y dopamina) no estaban relacionados con gingivitis determinadas con índice de sangrado gingival. Niveles de placa dental y número de superficies pro- ximales cariadas fueron significativamente asociadas con gingivitis; cuando factores demográficos y estresores psicosociales fueron añadidos al análisis, sólo la educación materna causó alguna asociación con gingivitis, sugiriendo para estos niños que el estrés emocional no sería un factor contribuyente al desarrollo de gingivitis.

13. (Glaser y cols 1999) (22). Diferentes estudios han observado que el estrés demora la cicatrización de las heridas. Este estudio valora la relación entre estrés psicológico y la secreción de citocinas proinflamatorias en heridas recientes.

Experimentó con 36 mujeres en las que se provocaba una ampolla en el antebrazo. Las mujeres con mayor estrés demostraron niveles significativamente más bajos de $\mathrm{IL}-1 \beta$ e IL-8 en las zonas de herida. Además, mujeres con bajos niveles de ambas citocinas después de 24 horas mostraron mayor estrés y niveles más altos de cortisol en saliva que aquellas con niveles altos de las anteriores citocinas.

14. (Suwatanapongched y cols 2000) (23). Estudio de la IL-1 $\beta$ en fluido crevicular de pacientes con periodontitis activa. Se observaron niveles incrementados en sitios con periodontitis activa, comparado con sitios inactivos o sanos. Este estudio evalúa la relación entre el nivel de IL-1 $\beta$ en fluido crevicular y el estatus de enfermedad periodontal.

Se tomaron muestras en 139 dientes de 26 sujetos (pacientes y controles). El nivel de IL-1 $\beta$ no mostró correlación con la profundidad de sondaje, pero tuvo correlación significativa con la extensión de pérdida de inserción.

Este estudio sugiere que el nivel de $\mathrm{IL}-1 \beta$ en fluido crevicular podría tener un valor predictivo para determinar el estatus periodontal activo o inactivo.

15. (Friedlander y cols 2001) (24). Estudio sobre los principales desórdenes depresivos que confiere valiosa información a los odontólogos sobre implicaciones clínicas del incremento de la prevalencia de esta enfermedad. Estos desequilibrios pueden producir: cambios de peso, fatiga, pérdida de concentración y deterioro físico. Estos individuos tienden a padecer comportamientos inadecuados, incluyendo mala higiene oral, dieta cariogénica, caries rampante, disminución del fluido salivar e incumplimiento de programas periodontales. La enfermedad periodontal avanzada normalmente es prevalente en estos individuos. Además, se ha observado que la medicación que toman estos pacientes para tratar los desórdenes psicológicos pueden producir xerostomía y así aumentar la incidencia de caries y enfermedad periodontal. 
16. (Hugoson y cols 2002) (25). Estudio de la relación entre algunos eventos negativos y factores psicológicos con respecto a la enfermedad periodontal en una población adulta, con edades comprendidas entre 50 y 80 años, en Suecia.

Observaciones clínicas y estudios epidemiológicos sugieren que experiencias negativas en la vida, especialmente aquellas manifestadas en forma de depresión, podrían contribuir a un aumento en la susceptibilidad a la enfermedad periodontal.

En este estudio se investigó la relación entre enfermedad periodontal, eventos desagradables en la vida y factores psicológicos. Estudio epidemiológico transversal realizado en 1993 en la ciudad de Jönköping (Suecia). Se seleccionaron randomizadamente 298 individuos dentados. Se registraron dientes existentes, índice de placa, índice gingival, profundidad de bolsa y hueso alveolar perdido. También se realizó un cuestionario sobre: estatus económico, acontecimientos vitales, factores psicológicos y factores de estrés.

Los resultados revelaron que, en adición a los bien documentados factores de riesgo periodontal como aumento de edad, higiene oral y tabaco; la pérdida de esposo/a (convirtiéndose en viudo/a), el tipo de personalidad y el control emocional también fueron asociados a enfermedad periodontal severa.

17. (Aleksejunienè y cols 2002) (26). Este estudio se realizó en 1997-98 en diez áreas de Lituania. Se recogió información sobre higiene oral, niveles de soporte periodontal e información sobre factores psicosociales y estilo de vida (en 681 sujetos).

Sólo el estilo de vida fue empíricamente demostrado como relacionado con el soporte periodontal. El estrés y el mantenimiento de salud periodontal no fueron empíricamente relacionados pero existen razones para pensar en una posible relación.

18. (Mengel y cols 2002) (3). El objetivo de este estudio fue el de medir los niveles en sangre periférica de IL-1 $\beta$, IL-6 y cortisol en pacientes con enfermedad periodontal para registrar alguna interacción con el estrés psicosocial.

- 16 pacientes sin tratar, con periodontitis agresiva generalizada (AGP).

- 14 pacientes tratados de periodontitis agresiva generalizada (AGP).

- 5 pacientes sin tratar, con periodontitis agresiva localizada (ALP).

- 5 pacientes sin tratar, con periodontitis crónica generalizada (CGP).

- 40 controles (salud periodontal).

IL-1 $\beta$, IL-6 y cortisol fueron medidos con el test de
ELISA. Se evaluó la profundidad de sondaje, la recesión gingival, el índice gingival, el índice de placa y el nivel clínico de inserción. Se utilizó un cuestionario para preguntar a los pacientes y controles sobre sus aptitudes en la vida y el estrés inducido por el trabajo y la familia. También se registró el nivel de consumo de tabaco.

Con respecto al cortisol, los resultados no mostraron importantes diferencias entre pacientes y controles. La IL-1 $\beta$ fue detectada sólo en pacientes con AGP y sus controles, pero sin importantes diferencias. La IL6 fue detectada en todos los pacientes pero sin importantes diferencias. Sólo en los no tratados, con AGP, la IL-6 estaba significativamente elevada y levemente correlacionada con pérdida de inserción. En todos los AGP, una leve correlación fue registrada entre IL- $1 \beta$ e IL-6. La evaluación de los cuestionarios reveló una alta proporción de pacientes no tratados con AGP con una actitud pesimista hacia la vida. En todos los AGP, la pérdida de inserción fue relacionada con estrés familiar y tabaco.

Este estudio no encontró correlación entre mediadores inmunológicos (IL-1 $\beta$, IL-6), glucocorticoides (cortisol) y los valores de estrés registrados. No obstante, los pacientes con AGP no tratada mostraron signos de actitud pesimista y un elevado nivel de IL-6 en sangre periférica. Según los autores, posiblemente el número de pacientes investigados fue muy pequeño para adecuar conclusiones.

19. (Pistorius y cols 2002) (27). Estudio sobre la relación entre factores del estrés y la enfermedad periodontal. Se realizaron cuestionarios en 120 pacientes con periodontitis crónica y en 122 controles (salud periodontal). Los resultados del estudio sugieren que los eventos estresantes podrían ejercer un efecto desfavorable en el curso de la periodontitis crónica.

\section{ABSTRACT}

In the chronic character of periodontitis, conditions or risk factors are implicated on the progression of this disease. Some examples are diabetes, tobacco, genetic disorders, etc. Recently, different studies have tried to make a relationship between the psychological stress and the progression of periodontal disease. These papers have had different results depended on the studied population. Several studies have investigated the molecular and cellular bases in the relationship between the immune system and the central nerve system. The aim of this article is to make a literature review of the association between the psychological stress and periodontal disease, studding the scientific evidence available of this association. 


\section{KEY WORLDS}

Stress, periodontal disease, aetiology, pathogenesis.

\section{CORRESPONDENCIA}

Barbieri Petrelli G.

Torcuato Fdez Miranda, 46. $2^{\circ}$ I

Tel: 985132618

E-mail: ger_bar@yahoo.com

\section{BIBLIOGRAFÍA}

1.Warchel S, Shebilske W. Psicología. Fundamentos y Aplicaciones. Quinta edición. Ed. Prentice Hall.

2. Olff M. Stress, depression and immunity: the role of defense and coping styles. Psychiatry Res 1999 Jan 18;85(1): 7-15.

3. Mengel R, Bacher M, Flores-de-Jacoby L. Interactions between strees, interleukin-1_, interleukin- 6 and cortisol in periodontally diseased patients. J Clin Periodontol 2002; 29: 1012-22.

4. García RI, Mhenshaw M, Krall EA. Relación entre la enfermedad periodontal y la salud sistémica. Periodontology 2000 (Ed Esp), Vol. 1, 2002, 21-36.

5. LeResche L, Dworkin SF. The role of stress in inflammatory disease: review of concepts and current findings. Periodontology 2000, Vol. 30, 2002, 91-103.

6. Bulut U, Develioglu H, Taner IL, Berker E. Interleukin-1 beta levels in gingival crevicular fluid in type 2 diabetes mellitus and adult periodontitis. J Oral Sci 2001 Sep;43(3): 171-7.

7. Shapira L, Frolov I, Halabi A, Ben-Nathan D. Experimental stress suppresses recruitment of macrophages but enhanced their P. gingivalis LPS-stimulated secretion of nitric oxide. J Periodontol 2000; 71: 476-81.

8. Shapira L, Houri-HaddadY, Frolov I, Halabi A, Ben Nathan D. The effect of stress on the inflammatory response to Porphiromonas gingivalis in a mouse subcutaneous chamber model. J Periodontol 1999: 70: 289-93.

9. Shannon L. Stress as a predisposing factor in necrotizing ulcerative gingivitis. J Periodontol 1969 Apr: 240-2.

10. Freeman R, Goss S. Stress measures as predictors of periodontal disease - a preliminary communication. Community Oral Dent Epidemiol 1993: 21: 176-7.
11. Moss ME, Beck JD, Kaplan BH, Offenbacher S, Weintraub JA, Koch GG, Genco RJ, Machtei EE, Tedesco LA. Exploratory case-control analysis of psychosocial factors and adult periodontitis. J Periodontol 1996: 67: 10609.

12. Croucher R, Marcenes WS, Torres MC, Hughes F, Sheiham A. The relationship between life-events and periodontitis: a case-control study. J Clin Periodontol 1997: 24: 3943.

13. Genco RJ, Ho AW, Kopman J, Grossi SG, Dunford RG, Tedesco LA. Models to evaluate the role of stress in periodontal disease. Ann Periodontol 1998: 3: 288-302.

14. Genco RJ, Ho WA, Grossi SG, Dunford RG, Tedesco LA. Relationship of stress, distress, and inadequate coping behaviors to periodontal disease. J Periodontol 1999; 70: 711-23.

15. Deinzer R, Forster P, Fuck L, Herforth A, Stiller-Winkler R, Idel $\mathrm{H}$. Increase of crevicular interleukin-1 beta under academic stress at experimental gingivitis sites and at sites of perfect oral hygiene. J Clin Periodontol 1999: 26: 1-8.

16. Deinzer R, Ruttermann S, Mobes O, Herforth A. Increase in gingival inflammation under academic stress. J Clin Periodontol 1998: 25: 431-3.

17. Deinzer R, Kottmann W, Föster P, Herforth A. After-effects of stress on crevicular interleukin-1b. J Clin Periodontol 2000: 27: 74-7.

18. Deinzer R, Hilpert D, Bach K, Schawacht M, Herforth $\AA$. Effects of academic stress on oral hygiene - a potential link between stress and plaque- associated disease? J Clin Periodontol 2001: 28: 459-64.

19. Axtelius B, Söderfeldt B, Nilsson A, Edwardsson S, Attström R. Therapy-resistant periodontitis. Psychosocial characteristics. J Clin Periodontol 1998: 25: 482-91.

20. Shizukuishi S, Hayashi N, Tamaga H. Lifestyle and periodontal health status of a Japanese factory workers. Ann Periodontol 1998; 3: 303-11.

21. Vanderas AP, Kavvadia K, Papagiannoulis L. Urinary catecholamine levels and gingivitis in children. J Periodontol 1998: 69: 554-60.

22. Glaser R, Kiecolt-Glaser JK, Marucha, PT, MacCallum RC, Laskowski B, Malarkey WB. Stress-related changes in proinflamatory cytokine production in wounds. Arch Gen Psychiatry, Volume 56(5). May 1999. 450-6.

23. Suwatanapongched P, Laohapand P, Surarit R, Ohmoto Y, Ruxrungtham K. Interleukin-l beta level in gingival crevi- 


\section{AVANCES}

Volumen 15 - No 2 - Agosto 2003

cular fluid of patients with active periodontitis. Asian Pac J Allergy Immunol 2000 Dec;18 (4).

24. Friedlander A, Mahler ME. Major depressive disorder. Psychopathology, medical management and dental implications. J Am Dent Assoc 2001: 132: 629-38.

25. Hugoson A, Ljungquist B, Breivik T. The relationship of some negative events and psychological factors to periodontal disease in an adult Swedish population 50 to
80 years of age. J Clin Periodontol 2002; 29: 247-53.

26. Aleksejunienè J, Holst D, Eriksen HM, Gjermo. Psychosocial stress, lifestyle and periodontal health. A hypothesised structural equation model. J Clin Periodontol 2002; 29: 326-35.

27. Pistorius A, Krahwinkel T, Willershausen B, Boekstegen C. Relationship between stress factors and periodontal disease. Eur J Med Res 2002 Sep 30; 7(9): 393-8. 